\title{
AVALIAÇÃO DO TEMPO DE JEJUM E SEDE NO PACIENTE CIRÚRGICO
}

\author{
THIRST AND FASTING TIME ASSESSMENT \\ IN SURGICAL PATIENTS
}

\section{EVALUACIÓN DEL TIEMPO DE AYUNO Y SED EN EL PACIENTE QUIRÚRGICO}

\author{
Isadora Pierotti ${ }^{1}$ \\ Thammy Gonçalves Nakaya ${ }^{2}$ \\ Aline Korki Arrabal Garcia ${ }^{1}$ \\ Leonel Alves do Nascimento \\ Marilia Ferrari Conchon ${ }^{1}$ \\ Ligia Fahl Fonseca ${ }^{3}$
}

Como citar este artigo: Pierotti I, Nakaya TG, Garcia AKA, Nascimento LA, Conchon MF, Fonseca LF. Avaliação do tempo de jejum e sede no paciente cirúrgico. Rev baiana enferm. 2018;32:e27679.

Objetivo: avaliar o tempo de jejum pré-operatório, presença e intensidade da sede no pós-operatório imediato. Método: pesquisa documental, quantitativa, descritiva, realizada em 2018, em hospital escola, com amostra de 749 pacientes. Resultados: o tempo médio de jejum absoluto foi de 15:00 (DP 6:30). Pacientes cirúrgicos da urologia apresentaram maior tempo de jejum (16:56; DP 9:09). Em relação à faixa etária, o tempo de jejum variou de 13:29 (DP 7:34) para crianças, a 15:06 (DP 6:32) em adultos e 15:41 (DP 4:37) em idosos. A sede foi um desconforto presente em 84,5\% dos pacientes, com intensidade média de 6,54 (DP 2,39) no pós-operatório. Houve associação significativa entre tempo de jejum e presença de sede. A maioria dos pacientes $(85,4 \%)$ não se queixou de sede espontaneamente. Conclusão: o tempo de jejum pré-operatório foi maior do que o preconizado, independente da clínica cirúrgica ou faixa etária. Os idosos apresentaram maior tempo médio de jejum.

Descritores: Jejum. Sede. Enfermagem Perioperatória.

Objective: to assess preoperative fasting time, presence and intensity of thirst in the immediate postoperative period. Method: documentary, quantitative, and descriptive research carried out in 2018, in a university bospital, with a sample of 749 patients. Results: the mean time of absolute dry fasting was 15:00 (SD 6:30). Urological surgery patients showed longer fasting time (16:56; SD 9:09). Regarding age group, the fasting time varied from 13:29 (SD 7:34) for children, 15:06 (SD 6:32) for adults, and 15:41 (SD 4:37) for elderly. Thirst discomfort was present in 84.5\% of the patients, with a mean intensity of 6.54 (SD 2.39) in the postoperative. There was a significant relationship between fasting time and the presence of thirst. Most patients (85.4\%) did not spontaneously complain about thirst. Conclusion: the preoperative fasting time was longer than expected, regardless of the surgical clinic or age group. The elderly group showed longer mean fasting time.

Descriptors: Fasting. Thirst. Perioperative Nursing.

\footnotetext{
Enfermeira(o). Mestre em Enfermagem. Londrina, Paraná, Brasil. leonel lan@hotmail.com

Enfermeira. Especialista em Cuidados Intensivos do Adulto. Londrina, Paraná, Brasil.

Enfermeira. Doutora em Enfermagem. Professora Associada do Departamento de Enfermagem da Universidade Estadual de Londrina. Londrina, Paraná, Brasil.
} 
Objetivo: evaluar tiempo de ayuno preoperatorio y presencia e intensidad de sed en posoperatorio inmediato. Método: estudio documental, cuantitativo, descriptivo, realizado en 2018 en hospital escuela, sobre muestra de 749 pacientes. Resultados: el tiempo promedio de ayuno absoluto fue de 15:00 (DS 6:30). Los pacientes quirúrgicos de urología presentaron mayor tiempo de ayuno (16:56; DS 9:09). Respecto al segmento etario, el tiempo de ayuno varió de 13:29 (DS 7:343) para niños a 15:06 (DS 6:32) en adultos y 15:41 (DS 4:37) en ancianos. La sed estuvo presente en $84,5 \%$ de los pacientes, con intensidad promedio de 6:54 (DS 2:39) en posoperatorio. Hubo asociación significativa entre tiempo de ayuno y existencia de sed. La mayoría de los pacientes $(85,4 \%)$ no se quejó espontáneamente de sed. Conclusión: el tiempo de ayuno preoperatorio fue mayor al recomendado, independientemente de la clinica quirúrgica o la faja etaria. Los ancianos mostraron mayor tiempo de ayuno.

Descriptores: Ayuno. Sed. Enfermeria Perioperatoria.

\section{Introdução}

O jejum pré-operatório é uma prática comum, recomendada com o propósito de garantir o esvaziamento gástrico e diminuir o risco de eventos respiratórios adversos. Estes ocorrem principalmente durante o procedimento anestésico, devido ao bloqueio dos reflexos protetores de vias aéreas $^{(1-2)}$. Todavia, o período de jejum pré-operatório observado na prática clínica excede o recomendado por protocolos bem estabelecidos, como o da Associação Americana de Anestesiologia (ASA) ${ }^{(3)}$ e Enhanced Recovery After Surgery (ERAS). Estas mudanças foram propostas por meio de protocolos multimodais, que utilizam medicina baseada em evidências para contribuir com a recuperação do paciente cirúrgico ${ }^{(4)}$.

O ERAS foi idealizado por um grupo multicêntrico europeu em 2001, e fundamentou a criação do Projeto Aceleração da Recuperação Total Pós-Operatória (ACERTO) implantado no Brasil em 2005. Constitui-se como um programa multidisciplinar de cuidados perioperatórios para acelerar a recuperação do paciente cirúrgico. Dentre seus pilares, encontra-se a redução do jejum no período pré-operatório ${ }^{(4)}$.

A ASA apresenta guidelines com altos níveis de evidência, e recomenda o consumo de líquidos claros sem resíduos em livre demanda até duas horas antes do procedimento anestésico cirúrgico. Incluem-se entre os líquidos sem resíduos: água, café (preto), chá, bebidas carbonatadas (refrigerantes), suco de frutas sem polpa, bebidas ricas em carboidratos. Em relação aos demais alimentos, recomenda quatro horas de jejum para leite materno, seis horas para dieta leve e leite não materno e oito horas para alimentos sólidos e gordurosos ${ }^{(3)}$.

A redução do tempo de jejum é fortemente recomendada, com a finalidade de acelerar a recuperação do paciente e reduzir o estresse cirúrgico. No entanto, na prática clínica, poucos serviços a adotam, submetendo seus clientes a períodos de jejum absolutos e extremamente prolongados $^{(5)}$.

Os efeitos do jejum prolongado potencializam as alterações metabólicas inerentes ao trauma cirúrgico. O organismo, após poucas horas sem ingerir nutrientes, reduz os níveis de insulina circulante e inicia processo de glicogenólise. Em pouco menos de 24 horas, o glicogênio hepático esgota-se. Concomitante ao processo da glicogenólise, a gliconeogênese também é ativada, utilizando proteína muscular como fonte de energia. A principal mudança fisiológica está relacionada com o aumento da resistência à insulina, influenciando na resposta imunológica e inflamatória, além do processo de cicatrização $^{(4,6)}$. Desse modo, o paciente em fase pré-cirúrgica encontra-se em catabolismo, com a finalidade de ofertar energia ao organismo para manutenção da homeostase celular ${ }^{(6)}$.

Adicionalmente, o jejum prolongado impacta de forma negativa não apenas no equilíbrio fisico, mas também em aspectos emocionais, além prejudicar a satisfação do paciente com a experiência cirúrgica. Um sintoma muito comum e de elevado desconforto relacionado ao jejum é a sede perioperatória ${ }^{(7)}$. O sintoma sede apresenta atributos desconfortáveis que afligem o paciente 
no Pós-Operatório Imediato (POI), como lábios, boca e garganta secas, língua e saliva grossa, gosto ruim na boca e vontade de beber água ${ }^{(8-10)}$.

Estudo realizado com pacientes cirúrgicos adultos e idosos apresentou média de jejum de 16,2 horas para líquidos claros e 17,3 horas para alimentos sólidos ${ }^{(10)}$. Em procedimentos infantis, essa realidade não é diferente, pois as crianças chegam a ser mantidas em jejum até 73 horas no pré-operatório $^{(11)}$. Outro estudo revelou tempo excessivo de jejum para $70 \%$ das crianças ${ }^{(12)}$.

Diversas pesquisas foram conduzidas com a finalidade de avaliar o tempo de jejum pré-operatório e sua influência na anestesia. Revisão da Cochrane, que reuniu 25 estudos com pacientes pediátricos, concluiu que as recomendações para ingestão de líquidos de até duas horas antes do procedimento cirúrgico é segura, uma vez que não houve diferença de pH e volume gástrico quantificados no intra-operatório ${ }^{(13)}$. Outros estudos com crianças também não encontraram correlação entre a diminuição do intervalo de jejum pré-operatório e o aumento do volume do conteúdo gástrico ${ }^{(13-15)}$. Isso acontece devido ao tempo de esvaziamento gástrico após a ingestão de líquido claro acontecer dentro de 30 minutos, em crianças saudáveis ${ }^{(15)}$. Além disso, não houve diferença estatistiticamente significante na alteração de pH e no volume gástrico residual, quando pesquisadores compararam uma versus duas horas de jejum ${ }^{(14)}$.

Diante do contexto dos tempos prolongados de jejum na prática clínica e as repercussões advindas desse evento e a fim de permitir subsídios para a tomada de decisão com enfoque no cuidado centrado no paciente, o presente estudo tem como objetivo avaliar o tempo de jejum pré-operatório, presença e intensidade da sede no pós-operatório imediato.

\section{Método}

Trata-se de uma pesquisa documental, quantitativa e descritiva. O Grupo de Estudo e Pesquisa em Sede (GPS) possui um banco de dados no qual são acrescentadas informações referentes às pesquisas realizadas. Os dados seguem o mesmo roteiro e protocolo de coleta, possibilitando, assim, análise posterior. A amostra foi composta por 749 pacientes cirúrgicos de ambos os sexos, submetidos à cirurgia eletiva ou de urgência/emergência entre janeiro de 2014 e abril de 2018.

O local de estudo foi um hospital universitário do Norte do estado do Paraná, de alta complexidade, com 316 leitos disponíveis para o Sistema Único de Saúde (SUS). Conta com um centro cirúrgico composto por sete salas operatórias, uma sala pré-anestésica e seis leitos em Sala de Recuperação Anestésica (SRA), realizando em média 640 cirurgias/mês. A coleta de dados foi realizada no POI, ainda na sala de recuperação anestésica.

O critério de inclusão foi ser paciente que permaneceu em POI em SRA, com idade acima de 4 anos, consciente e orientado em tempo e espaço e que capaz de autorrelatar o tempo que permaneceu em jejum pré-operatório. As informações referentes às crianças foram colhidas pelo relato do cuidador. Dessa amostra, foram excluídos os pacientes que apresentaram dor intensa ou dificuldade de comunicação, por não apresentarem condições adequadas para a participação em uma pesquisa.

$O$ roteiro de coleta de dados contemplou informações demográficas e variáveis relacionadas ao tempo de jejum pré-operatório, bem como a presença de sede, queixa espontânea e intensidade avaliada por uma escala verbal numérica (EVN) em adultos, com pontuação de 0 a 10, e a escala de faces (EF) na criança, com pontuação de 0 a 4 . A sede é um desconforto sistematicamente avaliado na instituição, quanto à presença e intensidade durante o período de recuperação anestésica.

Os dados foram duplamente digitados e tabulados no programa Microsoft Excel ${ }^{\circledR}$, recebendo tratamento estatístico descritivo com o auxílio do programa IBM SPSS Statistics 20. Realizou-se análise de medidas centrais (média e desvio padrão) para a idade, intensidade da sede e tempo de jejum pré-operatório. As variáveis sexo, clínica cirúrgica, presença de sede e verbalização espontânea foram descritas em números absolutos 
e frequências. A variável tempo de jejum apresentou distribuição não paramétrica, sendo utilizado o teste de Mann-Whitney para avaliar a associação do tempo de jejum na presença de sede.

Os dados analisados no presente estudo são secundários de pesquisas desenvolvidas pelo GPS, as quais obedeceram aos preceitos éticos estabelecidos na Resolução n. 466/2012 do Conselho Nacional de Saúde. Os estudos originais foram apreciados e receberam aprovação do Comitê de Ética em Pesquisa da Universidade Estadual de Londrina. Não houve identificação, nem contato individual com nenhum dos participantes.

\section{Resultados}

O banco de dados foi composto por 749 pacientes. Predominou o sexo feminino, com 417 (55,7\%) participantes, a idade média foi de 36,3 anos (DP 17,3), variando de 4 a 85 anos. A faixa etária foi subdividida em crianças (4-12 anos $\mathrm{n}=81)$, adultos (13-59 anos - n=586) e idosos (60-85 anos $-\mathrm{n}=82)$. As clínicas cirúrgicas, assim como o tempo médio de jejum, estão descritos na Tabela 1.

Tabela 1 - Distribuição descritiva do tempo absoluto em horas por clínica cirúrgica. Londrina, Paraná, Brasil - 2014-2018 (N=749)

\begin{tabular}{l|c|c|c|c|c}
\hline Clínica Cirúrgica & $\mathbf{n}$ & $\mathbf{\%}$ & $\begin{array}{c}\text { Tempo médio } \\
\text { de jejum }\end{array}$ & $\begin{array}{c}\text { Intervalo de } \\
\text { Confiança } \\
\text { (95\%) }\end{array}$ & $\begin{array}{c}\text { Desvio } \\
\text { Padrão }\end{array}$ \\
\hline Aparelho Digestivo & 50 & 6,7 & $15: 08$ & $13: 33-16: 43$ & $5: 33$ \\
Cabeça e Pescoço & 50 & 6,7 & $15: 19$ & $13: 44-16: 54$ & $5: 33$ \\
Cardíaca & 39 & 5,2 & $12: 56$ & $11: 25-14: 28$ & $4: 41$ \\
Ginecologia e Obstetrícia & 214 & 28,6 & $15: 03$ & $14: 12-15: 53$ & $6: 16$ \\
Neurocirurgia & 17 & 2,3 & $16: 37$ & $14: 52-18: 21$ & $3: 22$ \\
Oftalmologia & 15 & 2,0 & $13: 38$ & $10: 10-17: 05$ & $6: 15$ \\
Ortopedia & 179 & 23,9 & $15: 11$ & $14: 13-16: 08$ & $6: 31$ \\
Otorrinolaringologia & 31 & 4,1 & $15: 43$ & $14: 37-16: 49$ & $3: 00$ \\
Emergências Cirúrgicas & 16 & 2,1 & $16: 25$ & $11: 19-21: 31$ & $9: 34$ \\
Torácica & 9 & 1,2 & $14: 41$ & $10: 49-18: 32$ & $5: 01$ \\
Urologia & 48 & 6,4 & $16: 56$ & $14: 16-19: 35$ & $9: 09$ \\
Vascular & 16 & 2,1 & $13: 08$ & $10: 46-15: 29$ & $4: 26$ \\
Bucomaxilo & 16 & 2,1 & $14: 09$ & $12: 22-15: 56$ & $3: 20$ \\
Cirurgia Infantil & 47 & 6,3 & $13: 15$ & $10: 29-16: 02$ & $9: 27$ \\
Plástica & 1 & 0,1 & $14: 35^{*}$ & & - \\
Odontocirurgia & 1 & 0,1 & $11: 00^{*}$ & - & - \\
Total & 749 & 100 & $15: 00$ & $14: 32-15: 28$ & $6: 30$ \\
\hline
\end{tabular}

Fonte: Elaboração própria.

* Apresentaram apenas um paciente analisado.

Nota: Sinal convencional utilizado:

- Dado numérico igual a zero não resultante de arredondamento.

O tempo de jejum pré-operatório foi avaliado nas diferentes faixas etárias e estão descritos na Tabela 2.

Tabela 2 - Tempo de jejum absoluto em horas por faixa etária. Londrina, Paraná, Brasil - 2014-2018

\begin{tabular}{l|c|c|c|c|c}
\hline Faixa etária & $\mathbf{n}$ & $\mathbf{0}$ & Tempo médio de jejum & $\begin{array}{c}\text { Intervalo de } \\
\text { Confiança (95\%) }\end{array}$ & $\begin{array}{l}\text { Desvio } \\
\text { Padrão }\end{array}$ \\
\hline Crianças (4-12) & 81 & 10,8 & $13: 29$ & $11: 48-15: 09$ & $7: 34$ \\
Adultos (13-59) & 586 & 78,2 & $15: 06$ & $14: 35-15: 38$ & $6: 32$ \\
Idosos (60-85) & 82 & 10,9 & $15: 41$ & $14: 40-16: 42$ & $4: 37$ \\
Total & 749 & 100 & $15: 00$ & $14: 32-15: 28$ & $6: 30$ \\
\hline
\end{tabular}

Fonte: Elaboração própria 
A presença de sede foi relatada por 633 $(84,5 \%)$ pacientes. Apenas 109 (14,6\%) dos pacientes verbalizaram de forma espontânea este sintoma. Observou-se que a verbalização espontânea da sede foi maior em crianças $(56,8 \%)$, seguida pelos adultos (14,8\%) e idosos (6\%).

A média de intensidade da sede foi de 6,54 (DP 2,39). O tempo médio de jejum para os pacientes que apresentaram sede foi de 15:23 (DP 6:44) e dos pacientes que não apresentaram sede foi de 14:29 (DP 15:37). A intensidade média da sede nas crianças foi de 2,85 em uma escala de faces com variação de 0 a 4 .

O teste de Mann-Whitney mostrou que o tempo de jejum tem associação sobre a presença de sede $(U=25444,500 ; \mathrm{p}<0,001)$.

\section{Discussão}

A relevância deste estudo está em quantificar o descompasso entre o tempo de jejum pré-operatório preconizado e aquele praticado pelas instituições de saúde. Além disso, evidencia a presença e intensidade de um desconforto usualmente desconsiderado, apesar de prevalente: a sede. Os resultados encontrados neste estudo corroboram o observado na prática clínica e na literatura, de que o tempo excessivo de jejum pré-operatório vem se perpetuando na realidade da instituição em estudo, independente da clínica cirúrgica ou faixa etária do paciente.

Outras instituições públicas brasileiras registram dados semelhantes. Em ensaio clínico randomizado realizado com pacientes cirúrgicos idosos, foi encontrado um tempo médio de jejum absoluto de 13:30 horas. Este mesmo estudo mostrou que, na chegada do paciente no centro cirúrgico, $70 \%$ dos que estavam em jejum apresentavam fome e 90\%, sede. Além disso, o estudo evidenciou que apenas 40\% dos pacientes sentiram-se satisfeitos com o atendimento anestésico-cirúrgico, quando permaneceram em jejum absoluto. Este dado difere dos 90\% dos pacientes satisfeitos, que receberam bebida rica em carboidrato, duas horas antes do ato anestésico-cirúrgico ${ }^{(5)}$.
Até mesmo os próprios cirurgiões que prescrevem o jejum pré-operatório muitas vezes não se dão conta das horas excessivas registradas em suas prescrições. Em um estudo realizado com cirurgiões, foi-lhes perguntado quanto tempo de jejum pré-operatório era prescrito para sólidos e líquidos, e as respostas foram comparadas com os registros em prontuários. Os cirurgiões disseram prescrever oito horas para alimentos sólidos e três para líquidos. No entanto, o tempo médio de jejum registrado nos prontuários alcançou 12 horas (entre 8 a 21 horas) para alimentos sólidos e de dez horas (entre 2 a 18 horas) para líquidos claros ${ }^{(16)}$. Esses resultados são semelhantes ao do presente estudo. Essa pequena diferença de média entre jejum de alimentos sólidos e líquidos, como evidenciado no estudo citado, confirma que, na prática, as prescrições não são individualizadas de acordo com os diferentes tipos de alimento.

Ressalta-se que, neste estudo, o tempo médio de jejum dos idosos foi o mais alto, evidenciando o contraste existente entre o que é recomendado pelos protocolos e o realizado na prática. Dadas as características metabólicas desta população, os efeitos deletérios do jejum são potencializados, ocasionando o aumento de consumo das reservas metabólicas e exacerbando o trauma cirúrgico ${ }^{(4-5)}$.

Entre outros motivos do prolongamento do jejum está o atraso e as suspensões nas cirurgias, o que pode elevar o jejum até uma média de 16 horas $^{(17)}$. Este pode ter sido um dos motivos que explica a elevada média de tempo de jejum absoluto neste estudo.

A falta de individualização de prescrições para o jejum pré-operatório e o tempo excessivo sem se alimentar acarretam malefícios ao paciente. Dentre os principais, está o aumento do volume residual gástrico, com redução do $\mathrm{pH}$, aumentando o risco de broncoaspiração ${ }^{(6,13-14)}$. No entanto, a ingestão de líquidos claros, até duas horas antes do procedimento, torna o volume residual menor e o $\mathrm{pH}$ maior ${ }^{(17)}$. Somam-se a este argumento evidências de que o jejum pré-operatório prolongado, além de bastante desconfortável e desnecessário, pode ser prejudicial, 
ao potencializar ou perpetuar a resposta orgânica ao trauma cirúrgico ${ }^{(1,6,17)}$.

Entre os desconfortos deflagrados pelo jejum encontra-se a sede. Esta é definida como a busca por água e é apontada pelos pacientes cirúrgicos como um dos principais desconfortos experienciados nesse período. Está presente em $88,5 \%$ dos pacientes cirúrgicos pediátricos ${ }^{(11)} \mathrm{e}$ em $89,6 \%$ dos pacientes adultos ${ }^{(10)}$ em POI. No presente estudo, encontrou-se uma prevalência da sede em $84,5 \%$ dos pacientes, com associação significativa do tempo de jejum pré-operatório com a presença de sede no POI $(\mathrm{p}<0,001)$.

A sede é causada por uma confluência de fatores. Entre eles, no período pré-operatório, está ansiedade, medo do desconhecido, preparos inerentes ao procedimento a que será submetido, comorbidades pré-existentes, além do jejum prolongado e desnecessário. Como consequência de todos esses fatores, está o ressecamento da cavidade oral, que representa o principal e mais prevalente atributo da sede $\mathrm{e}^{(18-19)}$.

No transoperatório, o paciente é submetido a intubação orotraqueal, permanecendo com a cavidade oral exposta. Recebe fármacos anestésicos como opioides e anticolinérgicos, além de apresentar perda sanguínea ${ }^{(8,20)}$. Esses fatores contribuem para a ocorrência de reações bioquímicas e hormonais, desencadeando a sede. Esta é caracterizada por desconfortos intensos e identificada por atributos como: boca, lábios e garganta secos, língua e saliva grossas, gosto ruim na boca e vontade de beber água ${ }^{(9)}$.

No pós-operatório, esse paciente experiencia a face mais cruel da sede, ao permanecer em jejum por períodos demasiadamente prolongados, muitas vezes pela falta de conhecimento da equipe cirúrgica quanto à possibilidade de se manejar a sede de forma segura no perioperatório ${ }^{(21-22)}$. Este sintoma é citado pelos pacientes no pós-operatório como angustiante, sobrepujando a fome e até mesmo a dor. Não obstante, a equipe continua subvalorizando, submensurando e subtratando este sintoma ${ }^{(18,23-24)}$.

A sede perioperatória baseia-se na percepção individual e em sua verbalização. No presente estudo, observou-se que apenas 14,8\% dos adultos verbalizaram sede de forma espontânea, o que vem de encontro a evidência também produzida por este grupo de estudo, que demonstra o relato espontâneo da sede no POI apenas entre 13 a $18 \%$ dos pacientes ${ }^{(8)}$.

Dentre os principais fatores que colaboram para esse silêncio está a cultura institucionalizada de que o jejum deve ser mantido a qualquer custo, tanto no pré como no pós-operatório. Esse dogma é repetido e enfatizado para o paciente que, mesmo experienciando sede intensa durante todo o período perioperatório, mantém-se calado, considerando este o preço a ser pago para passar pela cirurgia com segurança ${ }^{(18,23)}$.

Em estudo de caso com um paciente que apresentou sede intensa durante o período pré, trans e pós-operatório imediato, mas não a verbalizou espontaneamente, identificou-se que ele considerou que a equipe que o assistia deveria tê-lo questionado a respeito, se fosse possível intervir no desconforto que sentia. Como não o fizeram, ele permaneceu em silêncio, prolongando, assim, seu sofrimento ${ }^{(23)}$.

O fato de a verbalização espontânea estar mais presente em crianças (59\% no presente estudo) sugere estar associada à espontaneidade presente nessa faixa etária ${ }^{(11,25)}$. Dessa forma, o silêncio em relação à sede não deve ser encarado como ausência do sintoma, mas, sim, um sinal a ser investigado ${ }^{(23)}$.

\section{Conclusão}

A média do tempo de jejum pré-operatório observado neste estudo foi de 15 horas, sendo maior no grupo de idosos (15:41). Pôde-se observar uma divergência importante do tempo de jejum preconizado por protocolos padronizados atualmente e o praticado pelo serviço de saúde em estudo.

Houve associação significativa entre o tempo de jejum pré-operatório e a presença de sede no pós-operatório. A sede foi um desconforto identificado na maioria dos pacientes $(84,5 \%)$, com intensidade elevada $(6,54)$. A verbalização espontânea foi pequena em adultos e idosos (14,8\% e 6\%), sendo maior nas crianças $(56,8 \%)$. 
Considerando os dados do estudo e as fortes evidências clínicas, reforça-se a importância de os serviços de saúde seguirem protocolos atuais, preconizados pelas associações de classe, realizando prescrições individualizadas para jejum de alimentos sólidos e líquidos. É essencial ainda que se compreenda o entendimento sobre os benefícios em receber líquidos claros duas horas antes do ato anestésico-cirúrgico. Além disso, na prática clínica, estratégias de manejo seguro da sede podem ser utilizadas por meio da identificação, mensuração e avaliação desse sintoma com intencionalidade. Essas ações visam minorar esse desconforto prevalente e desnecessário, aumentando a qualidade do cuidado ao paciente cirúrgico.

\section{Colaborações:}

1. concepção, projeto, análise e interpretação dos dados: Isadora Pierotti, Thammy Gonçalves Nakaya, Aline Korki Arrabal Garcia, Leonel Alves do Nascimento, Marilia Ferrari Conchon e Ligia Fahl Fonseca;

2. redação do artigo e revisão crítica relevante do conteúdo intelectual: Isadora Pierotti, Thammy Gonçalves Nakaya, Aline Korki Arrabal Garcia, Leonel Alves do Nascimento, Marilia Ferrari Conchon e Ligia Fahl Fonseca;

3. aprovação final da versão a ser publicada: Isadora Pierotti, Thammy Gonçalves Nakaya, Aline Korki Arrabal Garcia, Leonel Alves do Nascimento, Marilia Ferrari Conchon e Ligia Fahl Fonseca.

\section{Referências}

1. Andrew-Romit $\mathrm{J}$, Mortel $\mathrm{T}$ van de. Ritualistic preoperative fasting: is it still occurring and what can we do about it? ACORN J Perioper Nurs Aust [Internet]. 2011 Jan 1 [cited 2018 Jul 31];24(1):14-9. Available from: https://epubs.scu.edu.au/ hahs_pubs/1049

2. Frykholm P, Schindler E, Sümpelmann R, Walker R, Weiss M. Preoperative fasting in children: review of existing guidelines and recent developments. Br J Anaesth [Internet]. 2018 Mar [cited 2018 Jul
31];120(3):469-74. Available from: http://www. ncbi.nlm.nih.gov/pubmed/29452803

3. Practice Guidelines for Preoperative Fasting and the Use of Pharmacologic Agents to Reduce the Risk of Pulmonary Aspiration. Anesthesiology [Internet]. 2017 Mar [cited 2018 Aug 3];126(3):37693. Available from: http://www.ncbi.nlm.nih.gov/ pubmed/28045707

4. de-Aguilar-Nascimento JE, Salomão AB, Waitzberg DL, Dock-Nascimento DB, Correa MITD, Campos ACL, et al. ACERTO guidelines of perioperative nutritional interventions in elective general surgery. Rev Col Bras Cir [Internet]. 2017 Dec [cited 2018 Jul 31];44(6):633-48. Available from: http://www.ncbi. nlm.nih.gov/pubmed/29267561

5. Imbelloni LE, Nasiane Pombo IA, Morais Filho GB. Reduced fasting time improves comfort and satisfaction of elderly patients undergoing anesthesia for hip fracture. Brazilian J Anesthesiol (English Ed) [Internet]. 2015 Mar [cited 2018 Jul 31];65(2):117-23. Available from: http://linkinghub. elsevier.com/retrieve/pii/S0104001414001419

6. Aguilar-Nascimento JE, Perrone F, Assunção Prado LÍ. Jejum pré-operatório de 8 horas ou de 2 horas: o que revela a evidência? Rev Col Bras Cir [Internet]. 2009 Aug [cited 2018 Jul 25];36(4):350-2. Available from: http://www.scielo.br/scielo.php?script $=$ sci_ arttext\&pid $=$ S0100-69912009000400014\&lng $=$ pt\&t $\operatorname{lng}=\mathrm{pt}$

7. Carey SK, Conchin S, Bloomfield-Stone S. A qualitative study into the impact of fasting within a large tertiary hospital in Australia - the patients' perspective. J Clin Nurs [Internet]. 2015 Jul [cited 2018 Jul 31];24(13-14):1946-54. Available from: http://www.ncbi.nlm.nih.gov/pubmed/25959390

8. Conchon MF, Nascimento LA, Fonseca LF, Aroni P. Perioperative thirst: an analysis from the perspective of the Symptom Management Theory. Rev Esc Enferm USP [Internet]. 2015 Feb [cited 2018 Jul 25];49(1):122-8. Available from: http:// www.ncbi.nlm.nih.gov/pubmed/25789651

9. Martins PR, Fonseca LF, Rossetto EG. Developing and validating the Perioperative Thirst Discomfort Scale. Rev Esc Enferm USP [Internet]. 2017 Jul [cited 2018 Jul 25];51:e03240. Available from: http://www.scielo.br/scielo.php?script=sci_ arttext\&pid=S0080-62342017000100436\&lng=en\& tlng=en. doi: 10.1590/S1980-220X2016029003240

10. Pierotti I, Fracarolli IFL, Fonseca LF, Aroni P. Evaluation of the intensity and discomfort of 
perioperative thirst. Esc Anna Nery [Internet]. 2018 May 21 [cited 2018 Jul 25];22(3):11-9. Available from: http://www.scielo.br/scielo.php?script=sci_ arttext\&pid=S1414-81452018000300204\&lng=en\&t lng=en

11. Riviera AA. Prevalência e intensidade da sede infantil no pós-operatório imediato [dissertação]. [Internet]. Londrina: Universidade Estadual de Londrina; 2016 [cited 2018 Jul 25]. Available from: http://www.bibliotecadigital.uel.br/document/ ?code $=$ vtls000210646

12. Brunet-Wood K, Simons M, Evasiuk A, Mazurak V, Dicken B, Ridley D, et al. Surgical fasting guidelines in children: Are we putting them into practice? J Pediatr Surg [Internet]. 2016 Aug [cited 2018 Jul 31];51(8):1298-302. Available from: http:// www.ncbi.nlm.nih.gov/pubmed/27166876

13. Brady MCC, Kinn S, Ness V, O'Rourke K, Randhawa N, Stuart P. Preoperative fasting for preventing perioperative complications in children. Cochrane Database Syst Rev [Internet]. 2009 Oct 7 [cited 2018 Jul 31];(4):CD005285. Available from: http://www. ncbi.nlm.nih.gov/pubmed/19821343

14. Schmidt AR, Buehler P, Seglias L, Stark T, Brotschi $\mathrm{B}$, Renner T, et al. Gastric $\mathrm{pH}$ and residual volume after 1 and $2 \mathrm{~h}$ fasting time for clear fluids in children †. Br J Anaesth [Internet]. 2015 Mar [cited 2018 Jul 31];114(3):477-82. Available from: http:// www.ncbi.nlm.nih.gov/pubmed/25501720

15. Schmitz A, Kellenberger CJ, Liamlahi R, Studhalter M, Weiss M. Gastric emptying after overnight fasting and clear fluid intake: a prospective investigation using serial magnetic resonance imaging in healthy children t. Br J Anaesth [Internet]. 2011 Sep [cited 2018 Jul 31];107(3):425-9. Available from: http:// www.ncbi.nlm.nih.gov/pubmed/21676893

16. De-Marchi JJ, De-Souza MM, Salomão $\mathrm{AB}$, Nascimento JEA, Selleti AA, de-Albuquerque E, et al. Cuidados perioperatórios em cirurgia bariátrica no contexto do projeto ACERTO: realidade e o imaginário de cirurgiões em um hospital de Cuiabá. Rev Col Bras Cir [Internet]. 2017 Jun [cited 2018 Jul 31];44(3):270-7. Available from: http://www.ncbi. nlm.nih.gov/pubmed/28767803

17. Oliveira KGB, Balsan M, Oliveira SS, AguilarNascimento JE. A abreviação do jejum pré-operatório para duas horas com carboidratos aumenta o risco anestésico? Rev Bras Anestesiol [Internet]. 2009 Oct [cited 2018 Jul 31];59(5):57784. Available from: http://www.scielo.br/ scielo.php?script $=$ sci_arttext $\&$ pid $=S 0034-$ $70942009000500006 \& \operatorname{lng}=$ pt\&nrm=iso\&tln $\mathrm{g}=\mathrm{pt}$

18. Silva LCJR, Aroni P, Fonseca LF. Tenho sede! Vivência do paciente cirúrgico no período perioperatório. Rev SOBECC [Internet]. 2016 Sep 2 [cited 2018 Jul 25];21(2):75. Available from: https:// revista.sobecc.org.br/sobecc/article/view/21

19. Stevenson RJ, Mahmut M, Rooney K. Individual differences in the interoceptive states of hunger, fullness and thirst. Appetite [Internet]. 2015 Dec [cited 2018 Jul 25];95:44-57. Available from: http:// www.ncbi.nlm.nih.gov/pubmed/26119812

20. Zimmerman CA, Leib DE, Knight ZA. Neural circuits underlying thirst and fluid homeostasis. Nat Rev Neurosci [Internet]. 2017 Aug [cited 2018 Jul 25];18(8):459-69. Available from: http://www. ncbi.nlm.nih.gov/pubmed/28638120

21. do Nascimento LA, Fonseca LFLF, dos Santos CB. Inter-rater Reliability Testing of the Safety Protocol for Thirst Management. J Perianesth Nurs [Internet]. 2018 Aug [cited 2018 Ago 14];33(4):52736. Available from: https://www.sciencedirect. com/science/article/pii/S1089947216304269

22. Conchon MF, Fonseca LF. Efficacy of an Ice Popsicle on Thirst Management in the Immediate Postoperative Period: a Randomized Clinical Trial. J Perianesth Nurs [Internet]. 2018 Apr [cited 2018 Jul 25];33(2):153-61. Available from: https:// www.sciencedirect.com/science/article/pii/ S1089947216302489

23. Garcia AKA, Fonseca LF, Lodi CR. O silêncio que permeia a sede perioperatória: um estudo de caso [Internet]. Trabalho apresentado no XIII Congresso Brasileiro de Enfermagem em Centro Cirúgico, Recuperação Anestésica e Centro de Material e Esterilização. São Paulo; 2017. Anais (on-line). São Paulo; 2017 [cited 2018 Jul 25]. Available from: http://sobecc.tmeventos.com.br/anais2017/pdfs/ trabalho_2255.pdf

24. Dessotte CAM, Rodrigues HF, Furuya RK, Rossi LA, Dantas RAS. Estressores percebidos por pacientes no pós-operatório imediato de cirurgia cardíaca. Rev Bras Enferm [Internet]. 2016 jul-ago [cited 2018 Jul 25];69(4):741-50. Available from: http://www.scielo.br/scielo.php?script=sci arttext\&pid=S0034-71672016000400741\&lng=pt\&t $\operatorname{lng}=\mathrm{pt}$

25. Campana MC, Fahl Fonseca L, Lopes DFM, Martins PR. Percepção dos cuidadores quanto à sede 
Isadora Pierotti, Thammy Gonçalves Nakaya, Aline Korki Arrabal Garcia, Leonel Alves do Nascimento, Marilia Ferrari Conchon, Ligia Fabl Fonseca

da criança cirúrgica. Rev Rene [Internet]. 2015 nov-dez;16(6):799-808. Available from: http:// www.redalyc.org/articulo.oa?id=324043261005
Recebido: 16 de agosto de 2018

Aprovado: 11 de novembro de 2018

Publicado: 27 de dezembro de 2018

A Revista Baiana de Enfermagem utiliza a Licença Creative Commons - Atribuição-NãoComercial 4.0 Internacional. https://creativecommons.org/licenses/by-nc/4.0/ Este artigo é de acesso aberto distribuído sob os termos da Licença Creative Commons (CC BY-NC). Esta licença permite que outros remixem, adaptem e criem a partir do seu trabalho para fins não comerciais. Embora os novos trabalhos tenham de lhe atribuir o devido crédito e não possam ser usados para fins comerciais, os usuários não têm de licenciar esses trabalhos derivados sob os mesmos termos. 